\title{
Different Interference Effects in Musicians and a Control Group
}

\author{
Stefan Berti, ${ }^{1}$ Stefan Münzer, ${ }^{2}$ Erich Schröger, ${ }^{3}$ and Thomas Pechmann ${ }^{4}$ \\ ${ }^{1}$ Psychologisches Institut, Johannes Gutenberg-Universität Mainz, Germany \\ ${ }^{2}$ Fachbereich Psychologie, Universität des Saarlandes, Saarbrücken, Germany \\ ${ }^{3}$ Institut für Psychologie I, Universität Leipzig, Germany \\ ${ }^{4}$ Institut für Linguistik, Universität Leipzig, Germany
}

\begin{abstract}
In the present study musicians and normal control subjects performed an S1-S2 pitch comparison task, which included the presentation of intervening tones during the retention interval. The time for encoding and storing the pitch of S1 was varied between 200 and $1,500 \mathrm{~ms}$ by changing the pause between the S1 offset and the onset of the intervening tones. Although musicians outperformed the control group with longer pauses after the S1 offset, this advantage was relatively small with shorter pauses. These results suggest that the advantage of musicians in storing auditory information is not solely due to their superior encoding of information but also to improved working memory operations.
\end{abstract}

Keywords: audition, long-term memory, working memory, pitch comparison task, memory interference, distraction, musicians

\section{Introduction}

Interference effects in auditory short-term memory depend on a variety of factors. These can include the interference properties of separate stimuli (i.e., their similarity), the experience one has with a certain kind of material (i.e., the invocation of structures in long-term memory), or the attentional capacity or efficiency of processes that help to keep representations active and accessible (i.e., the effects of working memory). Following Cowan (1984), a distinction can be made between a short and long auditory store: the short auditory store is merely a literal trace lasting about $300 \mathrm{~ms}$ and is presumably an encoding process rather than a store, while the long auditory store lasts several seconds and can contain partly analyzed auditory features (cf. Winkler \& Cowan, 2005). The present article addresses the question of whether musicians (who have extensive training with tonal material) exhibit an advantage over a normal (untrained) population in maintaining tone representations against interference. More specifically, it is asked whether such an advantage occurs even during an early stage of auditory processing at the level of the short auditory store (during the first 100-300 ms), when a representation of a tone has to be built up by the auditory system.

Deutsch $(1970,1972 a, 1972 b, 1975,1982)$ developed a paradigm to test auditory short-term memory for a single tone frequency. In its original version, the paradigm required the comparison of two tones (S1 and S2, which were equal or different in frequency), separated by an interval of 5 seconds. In the experimental conditions, six intervening tones with different frequencies were placed within the 5 -second retention interval. In order to unravel the determinants of tonal interference, the pitch proximity of the intervening tones was varied relative to that of S1
(Deutsch, 1972b; Deutsch \& Feroe, 1975), or the repetition of the frequency of S1 was varied in the series of intervening tones (Deutsch, 1972a, 1975). From these studies it was concluded that short-term memory must consist of specialized, content-specific stores.

Pechmann \& Mohr (1992) compared musicians and nonmusicians using the tonal S1-S2 paradigm by Deutsch (1970) and found musicians to be less susceptible than nonmusicians to the interference effects caused by various types of stimuli (i.e., speech, visual, and tonal stimuli). In addition, Münzer \& Pechmann (2000) have demonstrated that the musician's advantage in preserving tonal codes is not due to a general enhancement of dual-task coordination but is restricted to tonal materials. From these studies it was concluded that the specific interference effects and the advantages of musicians over untrained control subjects relate to the use of specialized processes for tonal rehearsal in working memory - that is (presumably), at a processing stage when an internal representation of the stimulus already has been formed and may be kept active and protected against interference via specialized rehearsal routines.

The time frame used in Deutsch's paradigm allows 500 ms for every tone to be encoded. To examine more carefully the hypothetical role of working memory during tonal representation one may vary the stimulus-distractor interval to determine the functional role of relatively early (encoding) and relatively late (working memory) processing stages for the interference effects described above. From these studies one may conclude that (a) there are differences between musicians and subjects without special musical expertise in tonal interference effects in working memory dual-task performance, and (b) the encoding phase might critically influence interference effects. On this basis, the present experiment compared professional musi- 
cians and a control group with no extensive musical training in a shortened version of the tone comparison paradigm employed by Deutsch (1970). Tonal interference was introduced in the form of five intervening tones placed at different points in time within the interstimulus interval (i.e., 200, 300, $400 \ldots$. 1,500 ms after S1 onset). With this design the particular question was addressed as to whether interference effects depend on the time (early vs. late) of intervening-stimulus presentation following presentation of S1.

According to an event-related potentials (ERP) study by Koelsch, Schröger, \& Tervaniemi (1999) musicians may (under particular conditions) differ from nonmusicians in the processing of tones at around $150 \mathrm{~ms}$ after stimulus onset, suggesting superior encoding of musical information in musicians. However, even in conditions where such early effects are not present, ERPs may distinguish musicians from nonmusicians also at later stages of information processing, suggesting that musical expertise may exert its effects merely at attentive levels of processing rather than on preattentive encoding levels (Tervaniemi, Just, Koelsch, Widmann, \& Schröger, 2005). On the basis of this recent finding, it has to be expected that differences exist in the influence of the intervening tones on behavioral accuracy between musicians and controls in later phases, when interference is produced after some time given to encode the auditory stimulus and to form a memory representation. However, performance differences between musicians and controls in earlier phases, when the representation of the auditory stimulus is to be formed, seem plausible too.

In other words, we assume a two-stage model of the advantage of musical expertise on the processing of auditory information in distractor conditions. First, the encoding of information in sensory memory representations may be superior in musicians (Kölsch et al., 1999). Second, shielding against intervening sound during the retention interval (working memory operation) may be more effective in musicians than in controls (Münzer \& Pechmann, 2000; Pechmann \& Mohr, 1992; Tervaniemi et al., 2005). Thus, if the first stage contributes to the superior performance of musicians, then the improved encoding should result in an advantage for musicians that is observable within the first several hundred milliseconds. Additional benefits for musicians during later phases (beyond encoding) with increasing processing time would suggest that another process comes into play.

\section{Material and Method}

\section{Subjects}

Twenty-six students (13 men and 13 women, all between 18 and 26 years old) at the University of Leipzig and the Leipzig College of Music participated in this experiment. Eleven subjects ( 5 men and six women, all between 20 and 26 years old) were students from the Leipzig College of Music, including 9 instrumentalists (piano, flute, and violin) and 2 vocalists. They reported a starting age between 5 and 9 years (mean age 7 years) in their main instrument.
Moreover, 9 subjects reported to practice at least one additional instrument. These 11 students were assigned to the musician group. The remaining 15 participants $(8 \mathrm{men}$ and 7 women, all between 18 and 25 years old) were students of the University of Leipzig who had no special musical education and were assigned to the nonmusician control group. The subjects of the control group were not selected on the basis of a complete lack of musical experience. Half of these students had learned an instrument or were active singers several years previously, and one was practicing an instrument at the time the experiment was conducted. All subjects were paid for their participation. After the single subject data analysis, six students were excluded from the subsequent analysis for the following reasons: One subject from the musician group was excluded because of the ability of absolute pitch. Five subjects from the control group were excluded because they had too poor a performance in the control condition - that is, they had less than $80 \%$ correct responses in the easiest condition without intervening tones. The remaining participants in the musician group (4 men and 6 women, all between 20 and 26 years old) reported a starting age between 5 and 9 years (mean age 7.2 years), while in the control group ( 7 men and 3 women, all between 19 and 25 years old) 6 out of 10 participants reported some musical education as instrumentalists or singers.

\section{Stimulus Material}

The auditory material consisted of 15 sinusoidal tones with frequencies between 415 and $932 \mathrm{~Hz}$ in semitones (415, 440, 466, 494, 523, 554, 587, 622, 659, 698, 740, 784, $831,880,932 \mathrm{~Hz}$ ). Sinusoidal tones contain no additional musical information such as harmonic relations or the color of a sound. Therefore, any advantages that musicians might have are due to differential processing of mere frequency information. A tone lasted for $50 \mathrm{~ms}$ including 5-ms rise and fall times. From the 15 tones a subset of 11 tones (frequencies 466-831 Hz) was used in the S1-S2 comparison task. In the interference conditions there were five tones with different frequencies (from the set of 15 tones, $415-932 \mathrm{~Hz}$ ) that were presented as interpolated tones during the retention interval. All auditory events were presented binaurally with a sound pressure level of $75 \mathrm{~dB}$ (measured with an artificial head system HMS III.3) using a Sennheiser HD 25-1 headphone.

\section{Experimental Conditions}

Subjects performed the S1-S2 pitch comparison task in 11 separate, blocked conditions: 10 interference conditions and a control condition. The timing of the trials is depicted in Figure 1. In all conditions subjects were presented with an auditory stimulus as $\mathrm{S} 1$, followed by an interstimulus interval (ISI) of 3,150 ms. After this period (the retention interval), a second stimulus was presented as S2. S2 could be either of the same pitch as S1 or-with equal probability - S2 could be a semitone higher or lower than S1. With the onset of S2, the participant had to decide whether S1 and $\mathrm{S} 2$ had been of the same pitch. 
In the interference conditions (see Figures $1 \mathrm{~A}$ and $1 \mathrm{~B}$ for examples), five intervening tones were presented during the retention interval. The pitch of the intervening tones were close to the pitch of S1 but were not the same. In particular, the intervening tones were one semitone higher and lower, two semitones higher and lower, and-with equal probability — either three semitones higher or lower. The particular order of the presentation of these intervening tones was randomly determined in advance of the experiment. The intervening tones were separated by a fixed ISI of $150 \mathrm{~ms}$, while the time between the last intervening tone and S2 was varied according to the interference condition (see Figures 1A and 1B for examples). While the number, timing, and rule of selection of the intervening tones were the same, the conditions differed with respect to the interval between the offset of S1 and the onset of the first intervening tone (the stimulus-distractor interval). The shortest time delay between the offset of S1 and the onset of the first intervening tone was $150 \mathrm{~ms}$ (see Figure 1A). This condition served as the experimental control condition for the reason that the ISI of $150 \mathrm{~ms}$ is sufficient to perceive $\mathrm{S} 1$ as a single tone, but performance in the comparison task should be at chance level because it is difficult to perceive the particular pitch of S1 when an intervening tone follows immediately. In the other conditions, there was some additional interval that could be used to encode and store S1: the entire group of intervening tones was delayed following S1 offset by a fixed time interval (between 100 and 1,300 $\mathrm{ms}$ ) as compared with the 150-ms condition (see Figure 1B for an example). In this fashion, additional stimulus-distractor intervals of $250,350,450,550,650,750,850$, 1,150 , and $1,450 \mathrm{~ms}$ were realized.

Finally a control condition was employed with the same timing as the interference conditions but without the intervening tones during the retention interval (see Figure 1C). This condition served as the measure of the subjects' pure comparison performance in the absence of intervening events.

\section{Procedure}

Subjects sat in a sound-attenuated cabin (Industrial Acoustics Company) and were instructed to perform the pitch comparison task as quickly and as accurately as possible by pressing a keypad button (BeriSoft). Stimulus presentation and the acquisition of the subjects' reaction was controlled by an IBM-compatible PC (Intel 486) using the ERTS program package (BeriSoft). All subjects started with the control condition, after which the order of the interference conditions was randomly intermixed on a subject-by-subject basis. The experiment lasted about one hour.

\section{Data Analysis}

The mean $P_{\mathrm{r}}\left(P_{\mathrm{r}}=\right.$ hit-rate minus false-alarms $)$ and standard error of mean were calculated for each stimulus-distractor interval and the control condition separately for the musicians and the control group. $P_{\mathrm{r}}$ ranges from 0 (random

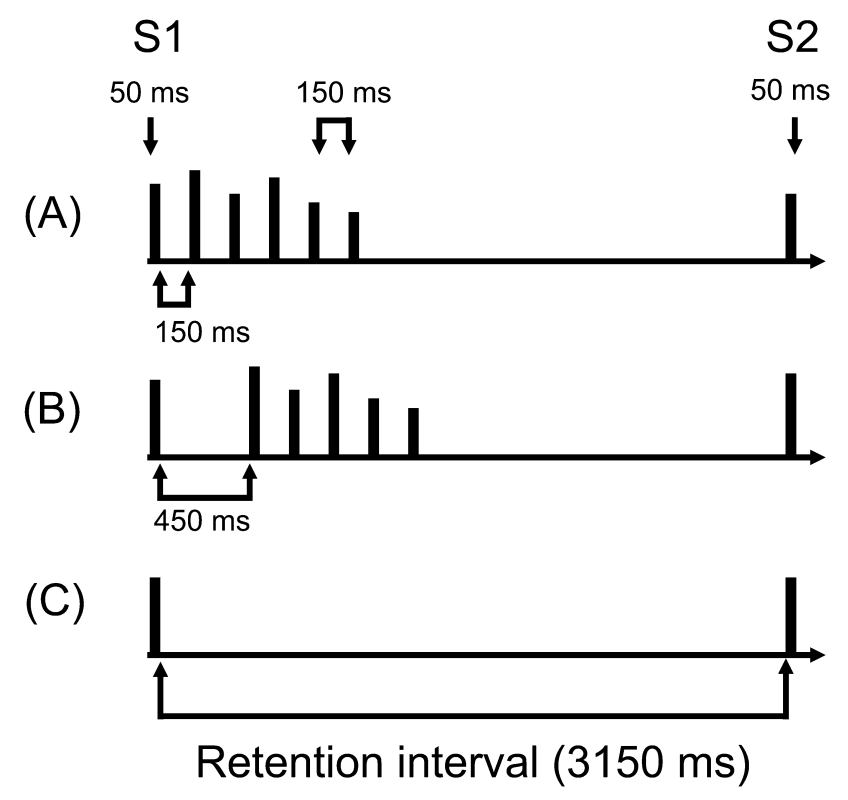

Figure 1. Timing of the S1-S2 task for 2 out of the 10 interference conditions, namely with a $150-\mathrm{ms}$ (A) and a 450-ms (B) stimulus-distractor interval, and the control condition $(\mathrm{C})$.

reactions) to 1 (perfect responses) and gives a measurement of true hits - that is, it is corrected for the probability of a lucky guess (estimated by the false-alarms, see Snodgrass $\&$ Corwin, 1988). $P_{\mathrm{r}}$ were analyzed by means of a mixed model analysis of variance (ANOVA) with the betweensubjects factor group (musicians vs. controls) and withinsubjects factor stimulus-distractor interval (10 interference conditions without the control condition). The HuynhFeldt correction was applied when needed. In case of a significant interaction, we computed subsequent statistical tests to unravel the nature of the interaction. In addition, the ANOVA was computed for the two groups separately to discern the effect of the stimulus-distractor interval factor within each group.

\section{Results}

The outcome of the experiment is illustrated in Figure 2. With a stimulus-distractor interval of $150 \mathrm{~ms}$, the performance of the participants in the control group is close to the performance of the musicians. In this condition, the processing of S1 seems to be disrupted irrespective of musical experience. In contrast, musicians seem to perform better than the control group in all other conditions.

The ANOVA revealed a main effect of the stimulusdistractor interval, $F(9,162)=14.33, p<.0001$, as well as a main effect of the group, $F(1,18)=5.89, p<.05$. These effects were qualified by a significant interaction, $F(9,162)=2.25, \mathrm{p}<.05$. Post-hoc nonparametric Wilcoxon tests revealed that musicians and participants in the control group did not differ significantly in recognition of the S2 tone in all stimulus-distractor interval conditions 


\section{Performance in the pitch comparison task}

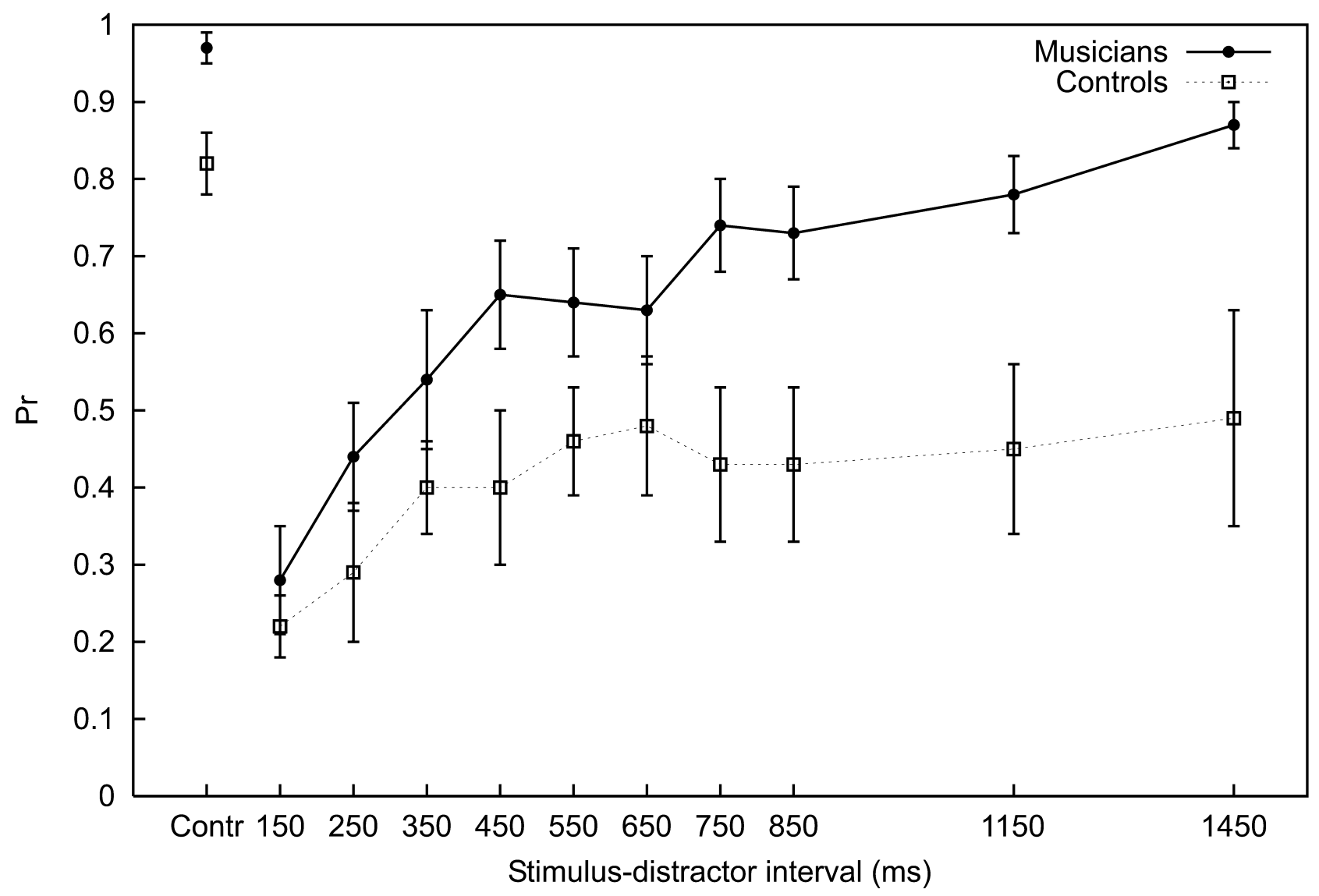

Figure 2. Performance (mean $P_{\mathrm{r}}$ and standard error of mean) of the musicians $(\bullet)$ and the control group $(\square)$ in the pitch comparison task under different interference conditions.

from 150 up to $650 \mathrm{~ms}(150 \mathrm{~ms}: p=.494 ; 250 \mathrm{~ms}: p=$ $.148 ; 350 \mathrm{~ms}: p=.128 ; 450 \mathrm{~ms}: p=.081 ; 550 \mathrm{~ms}: p=$ $.11 ; 650 \mathrm{~ms}: p=.255)$. In contrast, they differed in recognition of the S2 tone if the pause between S1 and the intervening tones was $750 \mathrm{~ms}$ or longer $(750 \mathrm{~ms}: p=.02$; $850 \mathrm{~ms}: p=.034$; $1,150 \mathrm{~ms}: p=.031 ; 1,450 \mathrm{~ms}: p=$ $.048)$. To test this late advantage of musicians more directly, $P_{\mathrm{r}}$ were collapsed over short (150-650 ms) and long (750-1,450 ms) stimulus-distractor interval conditions and were analyzed with an additional mixed-model 2 (musicians vs. controls) times 2 (short vs. long stimulus-distractor interval) ANOVA. This analysis mirrored the results of the 2 times 10 ANOVA: group: $F(1,18)=6.51, p<.05$; stimulus-distractor interval: $F(1,18)=28.24, p=.0001$; interaction: $F(1,18)=7.63, p<.05$. Again, post-hoc Wilcoxon tests showed a significant difference between musicians and controls for the long stimulus-distractor interval $(p=0.026)$ but not for the short interval ( $p=$ $0.121)$.

A subsequent one-way ANOVA for the stimulusdistractor interval factor was conducted for both groups separately. This effect was stronger for the musicians, $F(9$, $81)=15.83, p<.0001$, than for the controls, $F(9,81)=$ 2.92, $p<.05$. Subsequent planned contrasts (see Table 1 ) revealed that the performance in the 150 - and the $250-\mathrm{ms}$ stimulus-distractor interval condition differed in both groups compared with the performance in the 1,450-ms stimulus-distractor interval condition. In addition, within the musician group, other contrasts revealed significant effects, while in the control group, no further significant differences were obtained (Table 1). Moreover, an additional $t$ test for the control condition showed that musicians' and controls' performance in the pitch comparison task differ even when no interfering tones are presented during the retention interval, $t(18)=3.446, p<.01$.

\section{Discussion}

The results of the experiment demonstrate that musicians are superior in maintaining a tonal representation despite tonal interference. The significant interaction between the stimulus-distractor interval and the group factor shows that in musicians, the variation of the stimulus-distractor interval had a different effect than it had in the controls. Unambiguously, musicians outperformed the control group when the time to encode S1 was $750 \mathrm{~ms}$ or longer, while participants in the control group showed an impaired performance even with longest stimulus-distractor intervals. Since after $750 \mathrm{~ms}$ a representation of the tonal stimulus 
Table 1. Results of the statistical analysis separately for musicians and the control group: $F$ values of the planned contrasts of the one-way ANOVAs with the factor stimulus-distractor interval $(d f=1,9)$ and $p$ values.

\begin{tabular}{|c|c|c|c|c|c|c|c|c|c|}
\hline & \multicolumn{9}{|l|}{ Contrasts } \\
\hline & $\begin{array}{l}150 \mathrm{vs} . \\
1,450 \mathrm{~ms}\end{array}$ & $\begin{array}{l}250 \mathrm{vs} . \\
1,450 \mathrm{~ms}\end{array}$ & $\begin{array}{l}350 \mathrm{vs} . \\
1,450 \mathrm{~ms}\end{array}$ & $\begin{array}{l}450 \mathrm{vs} . \\
1,450 \mathrm{~ms}\end{array}$ & $\begin{array}{l}550 \mathrm{vs} . \\
1,450 \mathrm{~ms}\end{array}$ & $\begin{array}{l}650 \mathrm{vs} . \\
1,450 \mathrm{~ms}\end{array}$ & $\begin{array}{l}750 \text { vs. } \\
1,450 \mathrm{~ms}\end{array}$ & $\begin{array}{l}850 \text { vs. } \\
1,450 \mathrm{~ms}\end{array}$ & $\begin{array}{l}1,150 \mathrm{vs} . \\
1,450 \mathrm{~ms}\end{array}$ \\
\hline Musicians & $108.40 * * *$ & $73.63 * * *$ & $30.58 * * *$ & $15.12 * *$ & $12.66^{* *}$ & $17.94 * *$ & $6.30^{*}$ & $10.13^{*}$ & $6.38^{*}$ \\
\hline Controls & $5.46^{*}$ & $5.52 *$ & 0.57 & 1.01 & 0.06 & 0.04 & 0.33 & 0.84 & 0.50 \\
\hline
\end{tabular}

$* \mathrm{p}<.05 . * * \mathrm{p}<.01 . * * * \mathrm{p}<.001$.

should be established, this effect might be qualified as an index of different working memory processes. Thus, musicians and normal subjects without special musical expertise seem to differ in shielding an internal representation of a tonal stimulus against distracting tones. Importantly, these differences cannot be explained by an advantage of the musicians in processing special musical cues such as timbre or harmonic information because subjects had to perceive sinusoidal tones. It seems likely that musicians will perform above chance also with shorter stimulus-distractor intervals when presented with musical material such as chords. But such a result may overestimate the ability of musicians to encode and store the mere frequency information. Therefore, the advantage of musicians in this task must reflect a more general ability of musicians to keep tonal information.

In musicians, the auditory processing of a tonal stimulus was disrupted if other tonal stimuli follow immediately. This is demonstrated in the 150 -ms stimulus-distractor interval condition where there was no extra time given to build up a representation of S1. Musicians and control subjects performed with equal efficiency under these conditions. Furthermore, there was no significant difference between groups with short stimulus-distractor intervals of 250-750 ms after the S1 onset given for encoding. In the 450- and 550-ms stimulus-distractor interval conditions, there was a slight difference between musicians and the control group, but this difference disappeared with a stimulus-distractor interval of $650 \mathrm{~ms}$. Thus, although there was a numerical advantage for musicians with short stimulusdistractor intervals (supporting superior encoding of tonal information in musicians as claimed, e.g., by Koelsch et al., 1999), this difference was not statistically significant (being consistent with an absence of initial encoding as reported by Tervaniemi et al., 2005). Nevertheless, possibility of this small early advantage cannot explain the increase in the difference between musicians and the control group starting with stimulus-distractor intervals of $750 \mathrm{~ms}$.

That the groups benefit differently from longer stimulusdistractor intervals was revealed by separate analyses for both groups. In this respect, the performance of the control subjects benefits only from the first $100 \mathrm{~ms}$ prolongation of processing time (this is a total encoding time of $250 \mathrm{~ms}$ ). Contrary to the case for musicians, there is no additional increase in the performance of the control group if the stimulus-distractor intervals are longer. Noteworthy, longer stimulus-distractor intervals have an effect in participants in the control group, too: The increased variability with a 1,450-ms stimulus-distractor interval may reflect that some controls benefit from the longest stimulus-distractor interval while others do not. As noted above, we did not define the control group by a total lack of musical experience. But while all musicians benefit from the prolongation of the stimulus-distractor interval, only a few of the participants in the control group benefited from the longest stimulusdistractor interval. However, although subjects with musical interest were included, there is still a difference between the control group and musicians. On the other hand, an experiment reported by Deutsch (1982) shows that with a longer pause between S1 and the intervening tones, even nonmusicians may perform with a substantially reduced error rate in this task. In this experiment, nonmusicians made less than $10 \%$ errors with an encoding time of 6 seconds. Thus it seems that nonmusicians may behave like musicians when encoding time is increased well beyond $1,500 \mathrm{~ms}$.

In our opinion, this pattern of results could be explained within the model of Cowan (1988). In this model a short auditory store of around $300 \mathrm{~ms}$ is separated from a subsequent long auditory store of up to several seconds, which is the activated part of long-term memory. This reflects the distinction between sensory and short-term or working memory. It is assumed that the short auditory store is crucial for encoding the acoustic input into a neural representation available for subsequent processing (Cowan, 1984; Näätänen \& Winkler, 1999). In addition, working memory is defined as that part of activated long-term memory that is within the focus of attention. On these bases, one can distinguish between two stages of auditory processing: In the first stage, an internal representation of the tonal stimulus should be formed. This may happen during the first $300 \mathrm{~ms}$ after stimulus onset - the lifetime of the short auditory store. If tonal interference occurs during this first phase, the encoding process is interrupted and a representation cannot be properly established. In a second stage, an interference effect is assumed to occur that affects an already-formed internal representation, for instance in disturbing processes of maintaining the representation.

The additional improvement in musicians cannot be explained by processes on an encoding level. Therefore, the results of the present study suggest that musicians use processes or strategies to maintain tonal representation against interference within the context of working memory, which are not at the disposal of subjects with no special musical training or experience (see also Münzer \& Pechmann, 2000; Pechmann \& Mohr, 1992). For instance, it is well known that practice and the use of long-term structures have an important effect on the performance in short-term 
and working memory tasks (see, for instance, Ericsson \& Kintsch, 1995; Turner, Lucy, \& Smith, 2000). Therefore, it is possible that the advantage of musicians relies on specialized long-term structures built up during musical training and practice that could be activated more properly. Alternative explanations may be that musicians use the additional time to increase the activation of the memory content or that this time is sufficient to build a categorical representation in musicians upon the properly established memory trace. Those processes result in representations that are less affected by distracting events or could be rehearsed by the working memory system more effectively. In addition, it might be of interest whether different attentional capabilities may contribute to this difference (see Demany, Montandon, \& Semal, 2004, for the effect of selective attention on pitch retention).

In conclusion, the present data are compatible with the assumption of two (temporally overlapping) information processing stages: an early one of perceptual encoding of tonal information and a later one of maintenance of relevant information and shielding against irrelevant information in working memory. The existence of an early advantage has not only been shown in the musical domain (Koelsch et al., 1999), but also in other domains using the expert-novice approach. For example, chess experts show advantages for meaningful and, to some degree, also for random positions (Holding, 1985). The second stage is concerned with the maintenance of representations and their shielding against interference. These are two important functions of working memory, and the advantage of musicians may thus be qualified as a working memory advantage (Pechmann \& Mohr, 1992). The superior working memory functioning in musicians is very likely due to their long-term musical training and expertise. Thus, the present study shows again the importance of long-term memory for working memory processes as reflected in the model of Cowan (1988).

\section{Acknowledgments}

We would like to thank T. Ohlenbusch for her help in realizing the presented study and M. Elliott and B. Rosner for valuable comments on earlier versions of the article. This study was supported by the Working Memory Study Group of DFG (German Research Foundation) SCHR 375/ 8-2.

\section{References}

Cowan, N. (1984). On short and long auditory stores. Psychological Bulletin, 96(2), 341-370.

Cowan, N. (1988). Evolving concepts of memory storage, selective attention, and their mutual constraints within the human information processing system. Psychological Bulletin, 104(2), 163-191.

Demany, L., Montandon, G., \& Semal, C. (2004). Pitch perception and retention: Two cumulative benefits of selective attention. Memory \& Cognition, 66(4), 607-617.
Deutsch, D. (1970). Tones and numbers: Specificity of interference in immediate memory. Science, 168, 1604-1605.

Deutsch, D. (1972a). Effect of repetition of standard and comparison tones on recognition memory for pitch. Journal of Experimental Psychology, 93(1), 156-162.

Deutsch, D. (1972b). Mapping for interactions in the pitch memory store. Science, 175, 1020-1022.

Deutsch, D. (1975). Facilitation by repetition in recognition memory for tonal pitch. Memory \& Cognition, 3(3), 263-266.

Deutsch, D. (1982). The processing of pitch combinations. In D. Deutsch (Ed.), The Psychology of Music (pp. 271-316). New York: Academic Press.

Deutsch, D. \& Feroe, J. (1975). Disinhibition in pitch memory. Perception \& Psychophysics, 17(3), 320-324.

Ericsson, K. \& Kintsch, W. (1995). Long-term working memory. Psychological Review, 102(2), 211-245.

Holding, D. H. (1985). The psychology of chess skill. Hillsdale, NJ: Erlbaum.

Koelsch, S., Schröger, E., \& Tervaniemi, M. (1999). Superior preattentive auditory processing in musicians. Neuroreport, 10(6), 1309-1313.

Münzer, S. \& Pechmann, T. (2000). Concurrent processing of tonal and verbal materials in working memory: Do musicians differ from non-musicians? In E. Schröger, A. Mecklinger, \& A. Friederici (Eds.), Working on working memory (pp. 79-96). Leipzig, Germany: Leipziger Universitätsverlag.

Näätänen, R. \& Winkler, I. (1999). The concept of auditory stimulus representation in cognitive neuroscience. Psychological Bulletin, 125(6), 826-859.

Pechmann, T. \& Mohr, G. (1992). Interference in memory for tonal pitch: Implications for a working-memory model. Memory \& Cognition, 20(3), 314-320.

Snodgrass, J. G. \& Corwin, J. (1988). Pragmatics of measuring recognition memory: Applications to dementia and amnesia. Journal of Experimental Psychology: General, 117(1), 34-50.

Tervaniemi, M., Just, V., Koelsch, S., Widmann, A., \& Schröger, E. (2005). Pitch-discrimination accuracy in musicians vs. nonmusicians: An event-related brain potential and behavioral study. Experimental Brain Research, 161(1), 1-10.

Turner, J., Lucy, A., \& Smith, P. (2000). The development of the use of long-term knowledge to assist short-term recall. Quarterly Journal of Experimental Psychology, 53A(2), 457-478.

Winkler, I. \& Cowan, N. (2005). From sensory to long-term memory: Evidence from auditory memory reactivation studies. Experimental Psychology, 52(1), 3-20.

Stefan Berti

Psychologisches Institut

Johannes Gutenberg-Universität Mainz

Staudinger Weg 9

D-55099 Mainz

Germany

Tel. +4961313922889

Fax + 4961313922480

E-mail berti@uni-mainz.de 